\title{
Pilot Identification of Proposed Electronic Symbols for Displays of Aeronautical Charting Information
}

\author{
Divya C. Chandra and Michelle Yeh \\ United States Department of Transportation Volpe National Transportation Systems Center \\ Cambridge, Massachusetts \\ Colleen Donovan \\ Federal Aviation Administration \\ Washington, D.C.
}

\begin{abstract}
Many electronic displays of aeronautical charting information currently use different symbols for common display elements, creating the risk of confusion and misinterpretation. The SAE International Aerospace Behavior and Technology (G-10) Aeronautical Charting Committee, an industry group of subject matter experts, is developing an updated recommendations document that would provide guidance on what symbols to use on these displays. This paper describes a study conducted to evaluate some of the symbology proposed by the committee. Instrument-rated pilots were asked to identify proposed electronic symbols, and to rate their confidence in their response. The goal of this task was to determine whether pilots could correctly identify the proposed symbols, even though they may not be familiar with some of the specific symbols. Most of the symbols were well recognized, but a few were problematic.
\end{abstract}

\section{INTRODUCTION}

Electronic symbols for aeronautical charting information (e.g., navigation aids and instrument-approach symbols) are shown on many different flight-deck systems today. For example, some common navigation aid symbols and other aeronautical charting information, such as airspace boundaries, are typically shown on moving map displays. Electronic Flight Bags (EFBs) can also show charting information on electronic aeronautical charts, which are generally based on paper formats. (See Chandra, Yeh, Riley, \& Mangold, 2003 for a discussion of electronic charts on EFBs.) Database-driven electronic aeronautical charts that are intended to replace paper charts are also in development. These sophisticated charts will provide customizable electronic access to all information shown on paper charts, including reference values that may only be used occasionally (e.g., minimum altitudes for various situations).

The electronic symbols used on these displays sometimes vary significantly. Although there are industry-recommended standards for symbols on electronic displays of aeronautical charting information (International Civil Aviation

Organization [ICAO], 2001; RTCA, 2003; SAE, 1997), these standards are not always followed. In addition, some of the symbols that are in use currently are not well recognized by pilots (Yeh \& Chandra, 2006).

The lack of consistency in current symbology is not desirable from a human factors perspective. When different symbols are used to represent a single object or chart element, there is a risk of confusion. This is especially true because pilots could be using multiple sources of information at the same time (e.g., a moving-map display and a paper or electronic chart), and these sources may use different symbology conventions. In addition, there is the risk of conflicts when two manufacturers use the same symbol to represent different chart elements. Pilots could misinterpret a symbol if they rely upon knowledge of a different manufacturer's charts. The variety of symbology in use also impacts the cost of pilot training in the airlines, because airline operators need to ensure that pilots understand the symbology used on their standard-issue charts. Even pilots who rent aircraft are impacted because they may be exposed to different displays and symbologies with each rental.

Moving aeronautical charting symbology towards a higher level of standardization would help alleviate the problems noted above. In addition, standards would lower a manufacturer's development costs because the manufacturer would not have to design and test new symbols, and could be comfortable that the standard symbology would be approved by their Civil Aviation Authority (CAA).

With funding and support from the Federal Aviation Administration (FAA), the John A. Volpe National Transportation Systems Center (Volpe Center) is working with the SAE International Aerospace Behavior and Technology (G-10) Aeronautical Charting Committee in their efforts to update the SAE symbol recommendations (SAE, 1997). The industry committee consists of government and industry subject matter experts, including aeronautical chart and map display manufacturers, and pilots.

The Volpe Center's task is to determine whether pilots can correctly identify the committee's preliminary symbol proposals. The committee constructed the test symbol set based on symbols in use today. The proposed symbols may not be familiar to all pilots because they were taken from a variety of sources. In some cases, the committee was fairly confident in the proposal, but in others, the group was simply seeking more information about how pilots would respond to a symbol shape. The group intends to use the results of this study to help develop a final set of recommended symbols.

\section{METHOD}

The task at hand is to test the proposed recommended symbology for all electronic displays of charting information, including electronic aeronautical charts and moving-map displays. The symbols tested in this study include common 
display elements, such as obstructions, markers, and localizers. For more information on the symbols and how they are used in flight operations, see two FAA reference documents (2007a, 2007b).

The test was conducted in the form of a paper survey. Pilots were shown the symbol shape without any context, and asked to identify and write (i.e., recall) what the shape represented, and how confident they were in their response.

\section{Participants}

Ninety-six instrument rated pilots participated in the study. Almost all of the participants (84) held an Air Transport Pilot (ATP) rating. Their flight experience ranged from 800 to 25,000 flight hours, with an average of 8,300 flight hours. The participants had a variety of flight experience, though the majority (62) were active airline pilots. Seventeen pilots had military flight experience, and 37 had either private business or corporate flight experience. Many of the pilots had a mix of flight experience. For example, some airline pilots also had military experience.

Seventy-six pilots indicated that they primarily used charts from Jeppesen and 20 indicated they primarily used National Aeronautical Charting Office (NACO) charts, from the United States government. Ninety-one pilots indicated experience with glass cockpits, 90 with Flight Management Systems (FMS) displays, and 82 with moving-map displays.

\section{Procedure}

Pilots were recruited through the FAA, the Air Line Pilots Association (ALPA), the military (specifically, the Air Force Advanced Instrument School), and a local general aviation flying club. The study was advertised in a short verbal or written announcement (e.g., at a meeting or in a newsletter), and interested pilots were asked to contact the Volpe Center to have a paper questionnaire mailed to them. Participants did not receive any compensation for their participation.

Overall, 125 questionnaires were returned. Unfortunately, 29 of these had to be discarded, in most cases because the consent forms were not signed, leaving 96 useable responses. The bulk of the data came from responses to an electronic newsletter posting to ALPA members, which explains the high number of airline pilot participants. Only a few questionnaires were obtained from purely general aviation pilots, for whom the study was not broadly advertised. The overall response rate was not tracked.

Stimuli. Participants were presented with 16 proposed symbol shapes, listed alphabetically in Table 1 . Shapes for seven common navigation aids (the DME, intersection/fix, TACAN, VOR, VOR/DME, VORTAC, and waypoint) were tested earlier and agreed upon based on that study (Yeh \& Chandra, 2006), so they were excluded from the current study.

Note that two shapes were tested for three of the symbols, the generic localizer, MSA, and NDB. In these cases, the industry committee was seeking more information about how pilots interpret the symbols. Two foils (i.e., fake symbols) were also included (Table 2).

\begin{tabular}{|l|}
\hline \multicolumn{1}{|c|}{ Symbols Tested } \\
\hline Airport Beacon \\
\hline Back Course localizer \\
\hline Generic Localizer Candidates, 2 shapes tested \\
\hline Holding Pattern \\
\hline Locator Outer Marker (LOM) \\
\hline Marker (same shape for inner, middle, and outer marker) \\
\hline Minimum Safe Altitude (MSA), 2 shapes tested \\
\hline Multiple High Obstruction \\
\hline Multiple Low Obstruction \\
\hline Non-directional Beacon (NDB), 2 shapes tested \\
\hline Single High Obstruction \\
\hline Single Low Obstruction \\
\hline Terminal Arrival Area (TAA) \\
\hline
\end{tabular}

Table 1. Symbols tested.

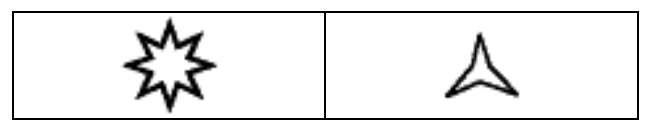

Table 2. Foils (i.e., fake symbols) included in the study.

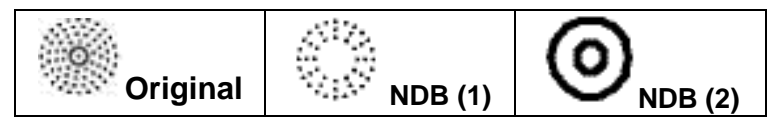

Table 3. NDB shapes.

The two candidates for generic localizer symbols (i.e., shapes that represent either a front or back course localizer) were based on variants of similar symbols in use today, which are shaped like long narrow arrowheads. One of the generic localizer shapes tested is currently used by NACO to represent a Simplified Directional Facility (SDF) or Localizer-Type Directional Aid (LDA) approach, which are uncommon procedures. The SAE G-10 Aeronautical Charting Committee was not sure whether that symbol was well known. If not, it could potentially be reused to indicate a generic localizer symbol. The second localizer shape had a similar outline as the first one, but included more detail.

Two MSA shapes were tested in order to obtain data to discriminate between reasonable alternatives: the current ICAO symbol, and the current NACO symbol.

The NDB symbol was included in this study because it was suspected that the original shape identified as stereotypical for an NDB in Yeh \& Chandra (2006), shown on the left in Table 3, could not be depicted easily on electronic displays. Therefore, a simplified version of the shape, NDB (1) in Table 3, was constructed and tested here. The second NDB shape in this study, NDB (2) in Table 3, was a double ring, which is used on some electronic displays. 
Task. Each symbol shape was shown in isolation. Participants were asked to identify the test symbol. Specifically, the instructions read:

For each shape below, identify it and indicate your level of confidence in your response. Some of the symbols are unusual, so you may not recognize all the symbols. Write “?” if you do not know or can't tell.

The second sentence was intended to reduce the chance that pilots would be frustrated by the inclusion of symbols that they did not recognize (i.e., the two foils). The expected response was a name/description for the symbol type (e.g., "group of obstacles").

\section{RESULTS}

Responses for the symbol identification task varied because participants could write in any response. In order to make sense of this variety, response categories were constructed for each symbol, based on the range of individual responses found for that symbol. Each individual response was then sorted into one of the response categories. For example, different individual responses for the obstruction symbol, such as "multiple towers" and "antennae" were both placed in one response category, called "man-made structure.” The response categories and response distributions for each symbol are detailed in Chandra \& Yeh (2007).

Tables showing the frequency of responses in each category were then constructed. These tables illustrate the range of responses, but by themselves, do not indicate whether the responses were “correct” or "acceptable.” In order to assess whether the responses were acceptable, a judgment call was made by the subject-matter-experts in the SAE G-10 Aeronautical Charting Committee. For example, the "manmade structure” response category was judged to be an acceptable response for the obstruction symbol, but the "terrain" response was not, because terrain is represented differently (e.g., by shaded contours). In some cases, however, acceptability of a response was not clear-cut. There were some responses that were understandable, but not technically correct. These situations are discussed in further detail below.

Results of the study are shown in Table 4 below, which shows the most well recognized symbols towards the top, and the most confusing symbols towards the bottom. Symbols that were recognized $70 \%$ of the time or better simply show the percent of correct responses as judged by the industry committee. Note that both MSA symbols were easily recognized. NDB (1), the simplified version of the NDB symbol from the earlier study (Yeh \& Chandra, 2006), was also well recognized.

Additional detail about peaks in the response distribution is presented for symbols that were less well recognized. Mean confidence ratings are also listed. Note that some of the localizer symbols were identified as representing an Instrument Landing System symbol, which is denoted "ILS" in Table 4.

\section{DISCUSSION}

Most of the symbols tested were identified correctly by a large number of pilots, generally $70 \%$ or better. At the borderline, with $65 \%$ correct recognition, is the back course localizer symbol. Responses to the last five symbols in Table 4 are the most varied relative to the others in the test. These shapes are the two candidates for a generic localizer, labeled Localizer (1) and Localizer (2), the airport beacon, the TAA, and NDB (2).

\section{Responses to Localizer Shapes}

A majority of participants (65\%) correctly identified the symbol shape representing a back-course localizer as a localizer. The second most common response for the backcourse localizer symbol shape was "ILS," given by $28 \%$ of pilots. The ILS response was considered to be incorrect by the SAE G-10 Aeronautical Charting Committee because the real ILS shape, which indicates availability of vertical guidance, is completely shaded or screened, not just shaded or screened on one side, as was the symbol tested in this study. However, the ILS symbol and the localizer symbol shapes do have a common outline. In addition, instrument approach charts with ILS procedures show a relatively large localizer symbol on the plan view (i.e., birds-eye view) of the chart, and they show a relatively smaller ILS symbol in the profile view of the chart, which depicts altitudes for the descent. Although incorrect, it is understandable that some pilots interpreted the localizer symbol to be an ILS symbol because of the prominence of the localizer shape on the instrument approach chart that is labeled as an ILS procedure.

Responses to the Localizer (1) and Localizer (2) shapes are still more varied. As noted earlier, the Localizer (1) shape is actually used as an SDF/LDA shape on NACO charts so, technically, just $24 \%$ of pilots responded correctly to that shape. In fact, this is a higher rate of recognition than the committee expected for such an uncommonly used symbol and procedure. On the other hand, $36 \%$ of pilots said they could not tell what the shape represented, so the symbol is not well recognized. Twenty-two percent of pilots responded that the Localizer (1) was a "localizer." This response was what the SAE G-10 Aeronautical Charting Committee thought that pilots might provide. However, given the high number of Can't Tell responses and significant number of SDF/LDA responses, this symbol appears to be confusing at best.

Responses to the Localizer (2) shape lean more favorably towards the anticipated localizer response (42\%). Fewer pilots confused this shape with an SDF/LDA symbol (10\%), or with an ILS symbol (10\%). In addition, there was a moderate rate of Can't Tell (17\%); this was better than the 36\% rate for Localizer (1), but worse than the $1 \%$ rate obtained for the back-course localizer shape.

There is also another, more positive, way to interpret the identification rates for the two generic localizer candidates. The rates of identification, while low relative to other more familiar symbols, could be seen as relatively good, considering that the symbols are new to pilots. This point of view assumes that pilots are highly attuned to all the details in a symbol, and the fact that they were able to extrapolate their knowledge to these different symbols was not necessarily expected. In other words, despite their novelty, the familiar outlines of the two localizer-symbol shapes did help some pilots to infer their meaning. 


\begin{tabular}{|c|c|c|c|}
\hline $\begin{array}{l}\text { Name of } \\
\text { Symbol }\end{array}$ & Symbol Shape & $\begin{array}{c}\text { Peaks in } \\
\text { Response } \\
\text { Distribution }\end{array}$ & $\begin{array}{c}\text { Mean } \\
\text { Confidence } \\
\text { Rating } \\
\text { (Max 7) }\end{array}$ \\
\hline $\begin{array}{l}\text { Holding } \\
\text { Pattern }\end{array}$ & & $\begin{array}{l}100 \% \\
\text { correct }\end{array}$ & 6.67 \\
\hline $\begin{array}{l}\text { MSA (1) } \\
\text { (ICAO } \\
\text { version) }\end{array}$ & & $\begin{array}{l}100 \% \\
\text { correct }\end{array}$ & 6.6 \\
\hline $\begin{array}{l}\text { MSA (2) } \\
\text { (NACO }\end{array}$ & & $97 \%$ correct & 6.37 \\
\hline $\begin{array}{l}\text { Multiple } \\
\text { High } \\
\text { Obstruction }\end{array}$ & & $95 \%$ correct & 6.19 \\
\hline Marker & & $90 \%$ correct & 6.00 \\
\hline $\begin{array}{l}\text { Single High } \\
\text { Obstruction }\end{array}$ & & $90 \%$ correct & 6.20 \\
\hline $\begin{array}{l}\text { Single Low } \\
\text { Obstruction }\end{array}$ & & $85 \%$ correct & 5.96 \\
\hline NDB (1) & & $83 \%$ correct & 6.01 \\
\hline $\begin{array}{l}\text { Multiple } \\
\text { Low } \\
\text { Obstruction }\end{array}$ & & $79 \%$ correct & 6.08 \\
\hline LOM & & $70 \%$ correct & 6.17 \\
\hline $\begin{array}{c}\text { Back } \\
\text { Course } \\
\text { Localizer }\end{array}$ & & $\begin{array}{c}65 \% \text { correct } \\
28 \% \text { ILS }\end{array}$ & 6.43 \\
\hline $\begin{array}{l}\text { Localizer } \\
\text { (2) }\end{array}$ & & $\begin{array}{c}42 \% \\
\text { Localizer } \\
10 \% \\
\text { SDF/LDA } \\
10 \% \\
\text { ILS } \\
17 \% \\
\text { Can't Tell }\end{array}$ & 4.91 \\
\hline
\end{tabular}

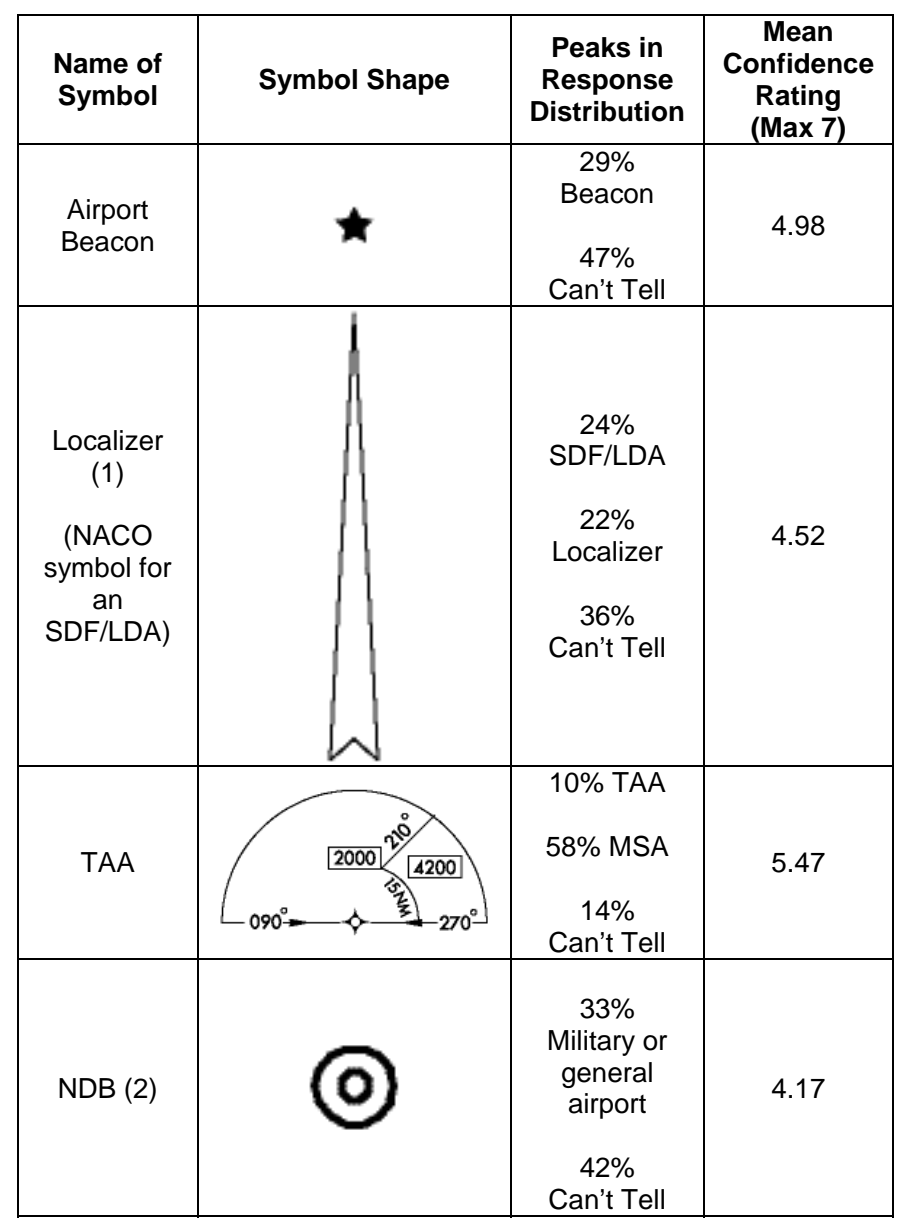

Table 4. Summary of results.

\section{Responses to Other Confusing Symbols}

NDB (2) is one of the shapes that received a variety of responses, although the most common response was "Can't Tell” (42\%). The shape was identified as either a military or generic airport by $33 \%$ of pilots. It was identified as an NDB by just $8 \%$ of pilots. In fact, the shape is very similar to that of a military airport (also a double circle), and somewhat similar to the generic airport symbol (a circle) that is used by some display manufacturers. The instructions did not say that airport symbols were excluded, so, without any other context, the airport response is not unreasonable. Although some transport display manufacturers do use this shape to represent an NDB, NDBs are rarely used in commercial operations, so the airline pilots in this study probably had little, if any, experience with this shape in that context. With an average rating of 4.17, pilot confidence was low for NDB (2), confirming its unfamiliarity.

The shape for a TAA was described correctly by just $10 \%$ of participants. The majority of pilots (58\%) described the shape as an MSA, which is visually similar to a TAA symbol. TAA's are used only on area navigation (RNAV) approaches so pilots may not be as familiar with them as with MSAs.

However, an MSA is, in fact, different from a TAA (see FAA, 2007a and 2007b). Both the TAA and MSA provide obstacle clearance, but in addition, a TAA provides altitude information for the transition from the enroute structure to the terminal area with minimal air traffic control communications. 
The star shape shown in Table 4, which was intended to represent an airport beacon, was described correctly as a beacon by only $29 \%$ of participants. A large number of participants (47\%) responded that they could not tell what the symbol represented. A few participants made responses that could be associated with the symbol shape (control tower, or facility not in continuous operation). Often, an airport symbol or label is shown next to the star shape to provide context. Without the symbol or label, the beacon symbol may have lost much of its meaning.

\section{Responses to the Foils}

The starburst-shaped foil was not identified by $86 \%$ of participants; $75 \%$ of pilots said “Can't Tell” and another $11 \%$ provided no response. Thirty-seven percent of participants did not identify the three-pointed star foil shape (32\% said “Can’t Tell and 5\% provided no response) but 53\% of the participants did call it a waypoint. In a previous study (Yeh \& Chandra, 2006) the stereotypical shape for a waypoint was identified as a four-pointed star; a three-pointed star, which was included as a foil in that study was not considered to be representative based on a statistical test.

\section{SUMMARY AND CONCLUSIONS}

The goal of the study was to provide information on whether pilots could identify several proposed symbol shapes that may be recommended in an industry recommendation document being drafted by the SAE G-10 Aeronautical Charting Committee. Most of the symbol-shapes tested were correctly identified by a majority of participants. A few problematic symbols were noted.

The problems encountered with these symbols can be discussed in the general context of two of the three ergonomic principles of design identified by Ben-Bassat and Shinar (2006), specifically familiarity and standardization. These two factors distinctly affect the recognition of the generic localizer and NDB shapes. Note that similarity across symbols may or may not be desirable, and should be considered carefully, because of the possibility of negative as well as positive transfer. In addition, familiarity of a symbol in the aeronautical environment is affected by frequency of exposure and use; symbols that are not often seen and used will be harder to recognize and may need additional labeling or contextual information. Symbol labels may give the pilot valuable clues for less familiar shapes.

It is important to emphasize that this research applies to any electronic display that shows these symbols, regardless of the intended function of display. The SAE G-10 Aeronautical Charting Committee will consider the results of this study as they develop an updated industry recommendations document. Results of this research are also intended to be of use to the FAA, industry, ICAO, and CAAs in general. These organizations may refer to the symbology recommendations developed by the SAE G-10 Aeronautical Charting Committee in their own guidance documents.

\section{ACKNOWLEDGMENTS}

This paper was prepared by the Human Factors Division of the Office of Aviation Programs at the Volpe Center, with funding from the FAA Human Factors Research and Engineering Group. We would like to thank our FAA program manager, Tom McCloy. We would also like to thank all the members of the SAE International G-10 Aeronautical Charting Committee, who invested their expertise and time to make the study more valuable. Particular thanks go to the many pilots who donated their time and input for the study. In addition, we would like to thank Catherine Guthy, Andrea Sparko, and Hao Tran for assistance with the data processing, and Raquel Rodriguez, for assistance with distributing the surveys.

The views expressed herein are those of the authors and do not necessarily reflect the views of the Volpe National Transportation Systems Center, the Research and Innovative Technology Administration, or the United States Department of Transportation.

\section{REFERENCES}

Ben-Bassat, T. \& Shinar, D. (2006). Ergonomic guidelines for traffic sign design increase sign comprehension. Human Factors, 48, 182-195.

Chandra, D. C., Yeh M., Riley, V. \& Mangold, S.J. (2003). Human factors considerations in the design and evaluation of Electronic Flight Bags (EFBs), Version 2 Cambridge, MA: USDOT Volpe National Transportation Systems Center. (NTIS No. PB2004101967)

Chandra, D.C. \& Yeh, M. (2007). Pilot identification of symbols and an exploration of symbol design issues for electronic displays of charting information (DOT/FAA/AR07/37, DOT-VNTSC-FAA-07-07). Cambridge, MA: USDOT Volpe National Transportation Systems Center.

Federal Aviation Administration (2007a). Instrument procedures handbook (FAA-H-8261-1A). Washington, DC: Author.

Federal Aviation Administration (2007b). Federal Aviation Regulations/Aeronautical information manual (ASA-07-FRAM-8K). Newcastle, WA: Aviation Supplies \& Academics.

International Civil Aviation Organization (ICAO) (July, 2001). Annex 4 to the convention on international civil aviation. Aeronautical charts. Montreal, Canada: Author.

RTCA (2003). Minimum operational performance standards for the depiction of navigational information on electronic maps. DO-257A. Washington, D.C.: Author.

SAE International (1997). Electronic aeronautical symbols, ARP 5289. Warrendale, PA: Author.

Yeh, M. \& Chandra, D. C. (2006). Pilot stereotypes for navigation symbols on electronic displays. In Reuzeau, F., Corker, K. \& Boy, G. (Eds.) Proceedings of the International Conference on Human-Computer Interaction in Aeronautics. (pp. 66-73). Toulouse, France: Cépaduès-Éditions. 